\title{
ProMode-Oligomer: Database of Normal Mode Analysis in Dihedral Angle Space for a Full-Atom System of Oligomeric Proteins
}

\author{
Hiroshi Wako*,a and Shigeru Endo ${ }^{\mathrm{b}}$ \\ ${ }^{a}$ School of Social Sciences, Waseda University, Tokyo 169-8050, Japan \\ ${ }^{b}$ Department of Physics, School of Science, Kitasato University, Sagamihara 252-0373, Japan
}

\begin{abstract}
The database ProMode-Oligomer (http://promode.socs.waseda.ac.jp/promode_oligomer) was constructed by collecting normal-mode-analysis (NMA) results for oligomeric proteins including protein-protein complexes. As in the ProMode database developed earlier for monomers and individual subunits of oligomers (Bioinformatics vol. 20, pp. 2035-2043, 2004), NMA was performed for a full-atom system using dihedral angles as independent variables, and we released the results (fluctuations of atoms, fluctuations of dihedral angles, correlations between atomic fluctuations, etc.). The vibrating oligomer is visualized by animation in an interactive molecular viewer for each of the 20 lowest-frequency normal modes. In addition, displacement vectors of constituent atoms for each normal mode were decomposed into two characteristic motions in individual subunits, i.e., internal and external (deformation and rigid-body movements of the individual subunits, respectively), and then the mutual movements of the subunits and the movement of atoms around the interface regions were investigated. These results released in ProMode-Oligomer are useful for characterizing oligomeric proteins from a dynamic point of view. The analyses are illustrated with immunoglobulin light- and heavy-chain variable domains bound to lysozyme and to a 12-residue peptide.
\end{abstract}

Keywords: Normal mode analysis, Oligomer, Internal motion, External motion, Immunoglobulin, Lysozyme.

\section{INTRODUCTION}

Protein folding and structure-function relationships are major challenges in molecular biology, biophysics, and other related fields. The three-dimensional structural data deposited in the Protein Data Bank (PDB) have played an important role in researching these problems. However, information extracted from the PDB contains mainly static structural features. It is generally recognized that both the dynamic and static aspects of protein structures are necessary to fully understand these problems. The only dynamics-related data provided in the PDB is temperature factor. This provides some information on the fluctuations of individual atoms, but also reflects crystal disorder. Since temperature factor is not sufficient for most problems on protein dynamics, it is necessary to perform computer simulations, such as molecular dynamics and normal mode analysis (NMA), to acquire more information about its dynamics from the PDB data.

NMA is a useful method for characterizing the dynamic aspects of biological macromolecules [1-5]. Normal modes of vibration are simple harmonic oscillations around an energy minimum and have various types of collective motions of atoms depending on their frequencies. Notably, it has been shown that some of the lower-frequency normal modes are strongly correlated with a large amplitude conformational change in proteins that has been observed upon ligand binding [6-13]. Furthermore, time-averaged properties, in particular mean-square fluctuation of an atom over all normal modes, have been proven to be well correlated with the tem

*Address correspondence to this author at the School of Social Sciences, Waseda University, Tokyo 169-8050, Japan; Tel: +81-3-5286-1430; Fax: +81-3-5272-0168; E-mail: wako@waseda.jp perature factor provided in the PDB data [1,2]. Protein flexibility prediction can also provide valuable information about a functional site [14-16].

Since the properties calculated from NMA are analytically well-defined, it is possible to systematically perform it for the data of many structures. Using this advantage, several databases of normal mode analysis of proteins have been developed to reveal their dynamic features [7,17-22]. ProMode is one such database developed by the authors [17]. ProMode is characterized by the use of dihedral angles as independent variables and its application to a full-atom system of monomeric proteins and individual subunits of oligomeric proteins. In this study, we extended the analysis to the oligomeric proteins, including protein-protein complexes, and developed an additional database named ProMode-Oligomer.

NMA has been applied to oligomeric proteins such as hemoglobin [23,24], G-protein coupled receptors [25], membrane proteins [26], and also complexes such as subtilisin-eglin c complexes [27] and protein-DNA complexes [21] to reveal structure-function relationships with particular attention to the influence of oligomerization and complex formation. In these studies, Ishida et al., [27] investigated the mutual motions between constituent molecules of the complex. In an NMA of an oligomeric protein (hereafter, since the same holds for both an oligomeric protein and a complex, we describe the process in terms of the oligomeric protein), translational and rotational motions of the whole protein are eliminated by the Eckart condition [28]. Accordingly, all motions considered are attributed to the internal degrees of freedom of the protein. However, when these internal motions are broken down further into motions of the 
constituent subunits, we have two motions characteristic to the oligomer, i.e., internal and external motions of individual subunits. The internal motion is a deformation motion, and the external one is a rigid-body motion of each subunit changing their mutual disposition. Since the external motion is considered key to understanding the dynamics of an oligomeric protein, information on internal and external motions of individual subunits are incorporated in ProModeOligomer. Since a simple formula for the decomposition of motions into the internal and external parts is possible in normal mode analysis in the dihedral angle space [27], we use this as a main characteristic feature of ProModeOligomer.

In this paper, we describe properties specific to oligomeric proteins released in ProMode-Oligomer, and provide examples of complexes of immunoglobulin light- and heavy-chain variable domains with lysozyme and with a 12residue peptide forming an $\alpha$-helix.

\section{METHOD}

Referring to the papers by Wako et al., [29] and Ishida et al., [27], we will briefly summarize the formulation for an oligomeric protein comprising two subunits. Extension to a protein consisting of three or more subunits is trivial. We use a molecular model with fixed bond lengths and bond angles. A local coordinate system is first defined for each subunit. We refer to the two local coordinate systems as systems I and II. A conformation of either subunit, i.e., a set of coordinate vectors of atoms, $\left\{r_{\alpha}\right\}$, is calculated using dihedral angles $\theta=\left\{\theta_{i}\right\}\left(i=1,2, \ldots, n_{j} ; n_{j}=n_{\mathrm{I}}\right.$ or $n_{\mathrm{II}}$ which is the total number of dihedral angles in systems I or II) as independent variables. Then, the coordinate vector of atom $\alpha$ in system II, $r_{\alpha}{ }_{\alpha}$, is transformed to the coordinate vectors in system $\mathrm{I}, r_{\alpha}{ }^{\mathrm{I}}$, by the following transformation:

$$
r_{\alpha}^{\mathrm{I}}=\tau+8 r_{\alpha}^{\mathrm{II}}
$$

where $\tau=\left(\tau_{1}, \tau_{2}, \tau_{3}\right)$ is the position vector of the origin of system II with respect to system I, and $\mathbf{U}=\left\{u_{\mu v}\right\}(\mu, v=1,2$, 3 ) is a rotation matrix that describes the relative orientation of system II to system I. The elements of $\mathbf{U}$ can be expressed by a rotation angle $s$ about a vector $\sigma=\left(\sigma_{1}, \sigma_{2}, \sigma_{3}\right)$ with a magnitude of $s$, i.e., $|\sigma|=s$, as follows:

$$
u_{\mu \nu}=s^{-2}(1-\cos s) \sigma_{\mu} \sigma_{v}-s^{-1} \sin s \sum_{\varsigma} \varepsilon_{\mu v \varsigma} \sigma_{\varsigma}+\delta_{\mu v} \cos s
$$

where if $\mu=v$, then $\delta_{\mu \nu}=1$, or else $\delta_{\mu \nu}=0$; and if $(\mu, v, \zeta)$ are even or odd permutations of $(x, y, z)$, then $\varepsilon_{\mu v \zeta}=1$ or -1 , respectively, or else $\varepsilon_{\mu v \zeta}=0$.

The conformational energy $E$ was calculated using ECEPP/3 force field [30] and minimized by FEDER/2 algorithm [29,31]. Energy minimization was performed with satisfactory accuracy such that the Hessian matrix, $\mathbf{F}=$ $\left\{\partial^{2} E / \partial \xi_{i} \partial \xi_{j}\right\}$, was positive definite at the energy minimum to reach, where $\xi_{i}=\theta_{i}, \tau_{i}$, or $\sigma_{i}$.

The normal modes of an oligomer are calculated by solving the generalized eigenvalue problem with the independent variables $\theta, \tau$, and $\sigma$ :

$\mathbf{F} \Omega=\mathbf{H} \Omega \Lambda$ with $\boldsymbol{\Omega}^{\mathbf{t}} \mathbf{H} \Omega=\mathbf{I}$
A coefficient matrix, $\mathbf{H}=\left\{H_{i j}\right\}$, for the kinetic energy is given as

$H_{i j}=\sum_{\alpha} m_{\alpha} \frac{\partial r_{\alpha}}{\partial \xi_{i}} \frac{\partial r_{\alpha}}{\partial \xi_{j}}$

where $m_{\alpha}$ and $r_{\alpha}$ are the mass and coordinate vectors of atom $\alpha$, respectively. $\boldsymbol{\Omega}=\left\{\omega_{i j}\right\}$ and $\mathbf{I}$ are the eigenvector and identity matrices, respectively. Matrix $\boldsymbol{\Lambda}$ is a diagonal matrix whose diagonal element is $\left(2 \pi v_{\lambda}\right)^{2}$, where $v_{\lambda}$ is a frequency of the $\lambda$ th normal mode. The $\lambda$ th normal mode variable is given as a harmonic oscillator of amplitude $A_{\lambda}$, frequency $v_{\lambda}$, and phase $\delta_{\lambda}$ :

$q_{\lambda}=A_{\lambda} \cos \left(2 \pi v_{\lambda} t+\delta_{\lambda}\right)$,

where

$A_{\lambda}=\frac{\sqrt{2 k_{\mathrm{B}} T}}{2 \pi v_{\lambda}}$,

$k_{\mathrm{B}}$ and $T$ are the Boltzmann factor and temperature, respectively. Temperature is set to $310 \mathrm{~K}$ in this study.

Displacement of the $b$ th variable $\xi_{b}$, i.e., a dihedral angle, a translational variable, or a rotational variable, is given with the normal mode variable $q_{\lambda}$ as

$\Delta \xi_{b}=\sum_{\lambda} \omega_{b \lambda} \Delta q_{\lambda}$ by

Then, the displacement vector $\Delta r_{\alpha}$ of atom $\alpha$ is calculated

$\Delta r_{\alpha}=\sum_{b}\left(\frac{\partial r_{\alpha}}{\partial \xi_{b}}\right) \Delta \xi_{b}=\sum_{\lambda} \Delta r_{\alpha, \lambda}$,

where

$\Delta r_{\alpha, \lambda}=\sum_{b}\left(\frac{\partial r_{\alpha}}{\partial \xi_{b}}\right) \omega_{b \lambda} \Delta q_{\lambda}$

$\Delta r_{\alpha}$ contains no contribution from the external degrees of freedom for the whole oligomer because $\left(\frac{\partial r_{\alpha}}{\partial \xi_{b}}\right)$ is calculated in such a way that the whole oligomer satisfies the Eckart condition [28].

Now, we break down $\Delta r_{\alpha}$ into internal and external motions. The internal displacement vector of atom $\alpha$ in subunit $k$ is given as

$\Delta r_{\alpha}^{\mathrm{int}}=\sum_{\lambda} \Delta r_{\alpha, \lambda}^{\mathrm{int}}=\sum_{\lambda} \sum_{b \in k}\left(\frac{\partial r_{\alpha}}{\partial \theta_{b}}\right)_{k} \omega_{b \lambda} \Delta q_{\lambda}$,

where $\left(\frac{\partial r_{\alpha}}{\partial \theta_{b}}\right)_{k}$ is calculated so as to satisfy the Eckart condition with respect only to subunit $k$. The second summation is taken only over dihedral angles in subunit $k$. The external displacement vector is defined as the difference between $\Delta r_{\alpha}$ and $\Delta r_{\alpha}^{\text {int: }}$

$\Delta r_{\alpha}^{e x t}=\Delta r_{\alpha}-\Delta r_{\alpha}^{\mathrm{int}}$ and $\Delta r_{\alpha, \lambda}^{e x t}=\Delta r_{\alpha, \lambda}-\Delta r_{\alpha, \lambda}^{\mathrm{int}}$. 
The time-averaged properties, e.g., three mean-square fluctuations of atom $\alpha$ : $\left\langle\left(\Delta r_{\alpha}\right)^{2}\right\rangle,\left\langle\left(\Delta r_{\alpha}{ }^{\text {int }}\right)^{2}\right\rangle$, and $\left\langle\left(\Delta r_{\alpha}{ }^{\text {ext }}\right)^{2}\right\rangle$, can be calculated with the help of the mean-square amplitude of the normal mode variables $\Delta q_{\lambda}$ as

$$
\left\langle\Delta q_{i} \Delta q_{j}\right\rangle=\frac{1}{2} \delta_{i j} A_{i},
$$

where $\delta_{i j}=1$ if $i=j$, and $\delta_{i j}=0$, otherwise. It should be noted that there is a residual term, i.e., a cross-correlation between $\Delta r_{\alpha}^{\text {int }}$ and $\Delta r_{\alpha}{ }^{\text {ext }}$,

$\left\langle\Delta r_{\alpha}^{\text {int }} \Delta r_{\alpha}^{\text {ext }}\right\rangle=\frac{1}{2}\left\{\left\langle\left(\Delta r_{\alpha}\right)^{2}\right\rangle-\left\langle\left(\Delta r_{\alpha}^{\text {int }}\right)^{2}\right\rangle-\left\langle\left(\Delta r_{\alpha}^{\text {ext }}\right)^{2}\right\rangle\right\}$,

in the relationship among $\left\langle\left(\Delta r_{\alpha}\right)^{2}\right\rangle,\left\langle\left(\Delta r_{\alpha}{ }^{\text {int }}\right)^{2}\right\rangle$, and $<\left(\Delta r_{\alpha}{ }^{\text {ext }}\right)^{2}>$, according to Eq. 10 .

Translational and rotational vectors for the external motion of the $\lambda$ th normal mode in subunit $k$ are defined as follows:

$$
\begin{aligned}
T_{k, \lambda} & =\frac{\sum_{\alpha \in k} m_{\alpha} \Delta r_{\alpha, \lambda}^{e x t}}{\sum_{\alpha \in k} m_{\alpha}} \\
R_{k, \lambda}= & \Gamma_{k}^{-1} \sum_{\alpha \in k} m_{\alpha}\left(r_{\alpha}^{0}-r_{k}^{G}\right) \times \Delta r_{\alpha, \lambda}^{e x t}
\end{aligned}
$$

where $r_{\alpha}{ }^{0}$ and $r_{k}^{G}$ are a coordinate vector of atom $\alpha$ in the minimum-energy conformation and the center of mass of subunit $k$, respectively. The summation is taken over the atoms in subunit $k$. The inertia tensor $\Gamma_{k}$ is given as

$\Gamma_{k}=\sum_{\alpha \in k} m_{\alpha}\left[\begin{array}{ccc}y_{\alpha}^{2}+z_{\alpha}^{2} & -x_{\alpha} y_{\alpha} & -x_{\alpha} z_{\alpha} \\ -x_{\alpha} y_{\alpha} & z_{\alpha}^{2}+x_{\alpha}^{2} & -y_{\alpha} z_{\alpha} \\ -x_{\alpha} z_{\alpha} & -y_{\alpha} z_{\alpha} & x_{\alpha}^{2}+y_{\alpha}^{2}\end{array}\right]$,

where $\left(x_{\alpha}, y_{\alpha}, z_{\alpha}\right)=\left(r_{\alpha}^{0}-r_{k}^{G}\right)$. The dimensions of the two vectors, $T_{k, \lambda}$ and $R_{k, \lambda}$, are $\AA$ and radians, respectively. The two vectors are useful for examining mutual movements of subunits.

\section{RESULTS AND DISCUSSION}

\subsection{Database contents}

The NMA results for more than 200 oligomers including protein-protein complexes have been released in ProModeOligomer, which is available at http://promode.socs.waseda. ac.jp/promode_oligomer. Since conformational energy minimization is time consuming, particularly for large proteins in NMA, the available oligomer proteins are limited to protein oligomers with less than 500 amino acid residues, and consequently the number of data entries is not very large.

The following data are provided for each oligomer in ProMode-Oligomer (Fig. 1):

(1) Time-averaged properties

i) Total fluctuations of atoms in the whole oligomer and internal and external fluctuations in individual subunits averaged over atoms in every residue and plotted against residue number.

ii) Fluctuations of main-chain dihedral angles $\varphi$ and $\psi$ and a side-chain dihedral angle $\chi^{1}$ plotted against residue number.

iii) Correlations between total fluctuations of $\mathrm{C}^{\alpha}$ atoms in the whole oligomer, which are represented in a triangular map.

$i v$ ) Inner products of internal and external displacement vectors of atoms defined in Eq. 12, which are given in the temperature factor column of a PDB-format data file. Residues with large negative values are indicated by color in the 3D structure on a molecular viewer.

(2) Properties related to each normal mode (for the 20 lowest-frequency modes)

i) Magnitudes of the displacement vectors of atoms for total fluctuations in the whole oligomer and for internal and external fluctuations in individual subunits, which are averaged over atoms in every residue and plotted against residue number.

ii) Fluctuations of main-chain dihedral angles $\varphi$ and $\psi$ and a side-chain dihedral angle $\chi^{1}$ plotted against residue number.

iii) Correlations (inner products) between displacement vectors of $\mathrm{C}^{\alpha}$ atoms in the whole oligomer, which are represented in a triangular map.

iv) Animation of the vibrating oligomer on a molecular viewer.

v) Displacement vectors of atoms for total fluctuation in the whole oligomer and for internal and external fluctuations in individual subunits defined in Eqs. 8-10, and translational and rotational vectors for the external motion defined in Eqs. 13 and 14. They are displayed on a molecular viewer and magnified for clarification. Data only for the 3 lowest-frequency normal modes are available.

vi) Inner products of internal and external displacement vectors of atoms defined in Eq. 12, which are given in the temperature factor column of a PDB-format data file. Residues with large negative values are indicated by color in the 3D structure on a molecular viewer.

A brief comment is necessary on 1-iv and 2-vi. Atoms with a negative inner product of internal and external displacement vectors are predominantly found at the interface of subunits. This means that the external motion has an almost inverse phase with the internal motion to attenuate the total motion. This is an important characteristic of oligomer interfaces. In ProMode-Oligomer, the atoms with a negative inner product are indicated in the three-dimensional structure by color on a molecular viewer, and thus the interface region is suggested from a dynamic perspective.

In addition, it is possible to compare the monomeric and oligomeric states of the oligomers because the NMA results for individual subunits are also provided in ProMode (http://promode.socs.waseda.ac.jp/). 


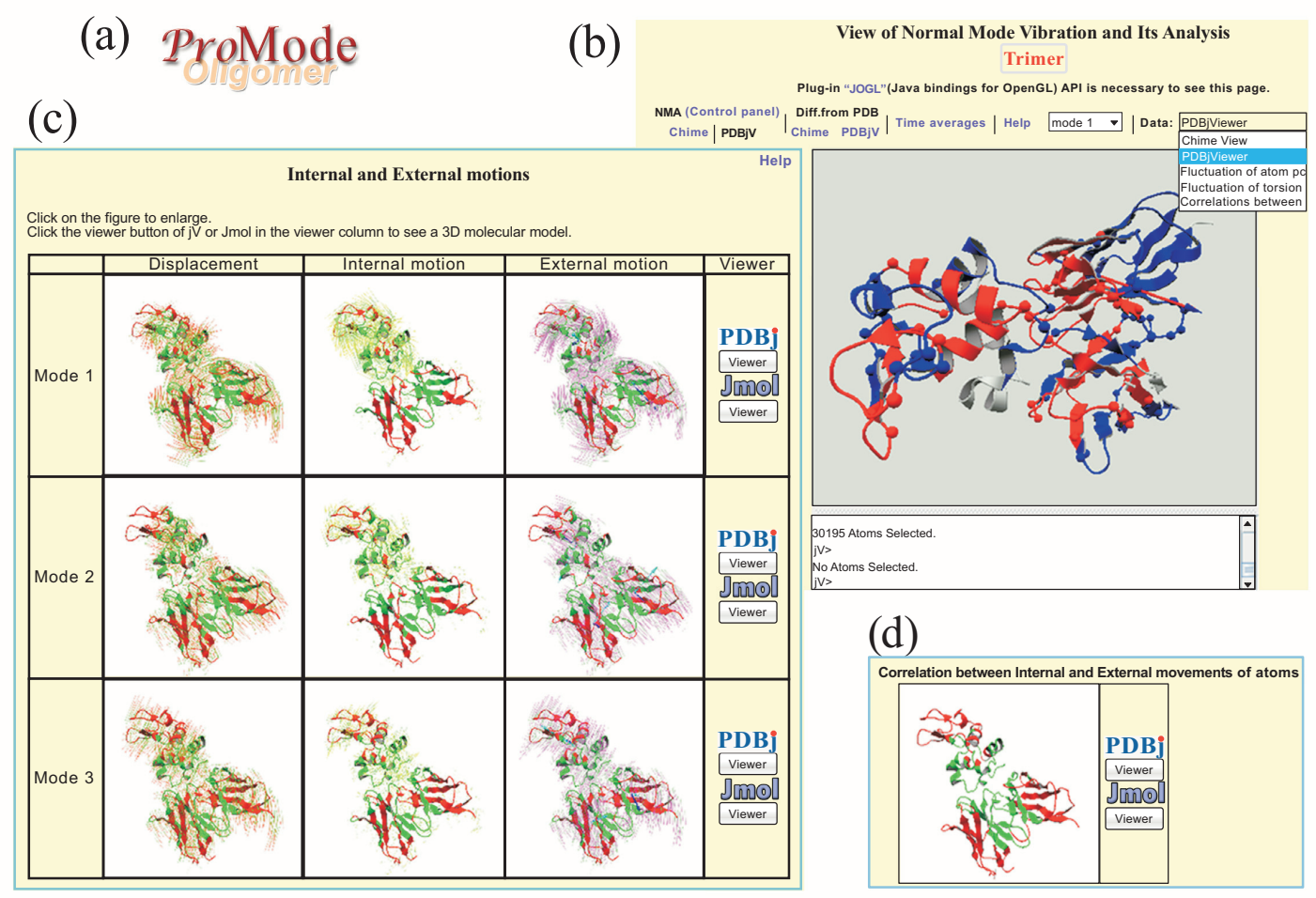

Fig. (1). Snapshots of ProMode-Oligomer. (a) The logo of ProMode-Oligomer. (b) Animation of a vibrating oligomer for each of the 20 lowest-frequency modes and properties associated with it, and time-averaged properties, (c) displacement vectors of atoms for total fluctuation in the whole oligomer and for internal and external fluctuations in individual subunits, and translational and rotational vectors for the external motion in individual subunits for the 3 lowest-frequency modes, (d) a color-coded 3D-structure according to inner products of internal and external displacement vectors of atoms, are presented in the ProMode-Oligomer page (see text for detail).

\subsection{Examples}

We illustrate the analysis of oligomeric proteins by two complexes of $\mathrm{Fv}$ fragments, i.e., dimers of immunoglobulin (Ig) light- and heavy-chain variable domains with lysozyme: 1bvk comprising chains A, B, and C [32], 2dqj comprising chains $\mathrm{L}, \mathrm{H}$, and $\mathrm{Y}$ [33], and a complex of the Fv fragment with a 12-residue peptide $1 \mathrm{p} 4 \mathrm{~b}$ comprising chains $\mathrm{L}, \mathrm{H}$, and $P$ [34], where the three complexes are referred to by their PDB code names. Lysozyme binds to the Fv fragment in different manners in 1bvk and 2dqj.

The structural alignments of the three complexes are shown in Fig. (2). There are four kinds of chains: Ig light chains (1bvkA, 2dqjL, and 1p4bL), Ig heavy chains (1bvkB, $2 \mathrm{dqj} H$, and $1 \mathrm{p} 4 \mathrm{bH}$ ), lysozyme (1bvkC and 2dqjY), and a 12residue peptide $(1 \mathrm{p} 4 \mathrm{bP})$. The structural alignments are performed separately for Ig light and heavy chains on the MATRAS web site [35]. Lysozyme (1bvkC and 2dqjY) has an identical amino-acid sequence. The peptide (1p4bP) does not have any counterpart to be aligned with.

Fig. (3a) shows the root-mean-square (RMS) total fluctuation of atom $\alpha$ of the complex, $\left\langle\Delta r_{\alpha}^{2}\right\rangle^{1 / 2}$. Figs. (3b and $\mathbf{3 c}$ ) show the RMS internal and external fluctuations, $\left\langle\left(\Delta r_{\alpha}^{\text {int }}\right)^{2}\right\rangle^{1 / 2}$ and $\left\langle\left(\Delta r_{\alpha}^{e x t}\right)^{2}\right\rangle^{1 / 2}$, respectively. The RMS value averaged over atoms in each residue is plotted against the residue number assigned based on the structural alignment in Fig. (2). In addition, cross-correlation between internal and external motions, $\left\langle\Delta r_{\alpha}^{\text {int }} \Delta r_{\alpha}^{\text {ext }}\right\rangle$, defined by Eq. 12 is shown in Fig. (3d).

In Fig. (4), displacement vectors of internal and external fluctuations of individual subunits are shown together with the total fluctuation of the complex for the lowest-frequency normal mode of 2dqj. Data not shown here are available at ProMode-Oligomer.

The fluctuations of the Fv fragments in the three complexes have similar features in the total and internal fluctuations of atoms (Figs. 3a and 3b). However, significant differences are observed in the external fluctuations (Fig. 3c). The external fluctuation that originates rigid-body motions of Ig light- and heavy-chains is largest in 1bvk and smallest in 1p4b. Since the Fv fragments in 1p4b bind much smaller polypeptide than lysozyme, they behave like a single-chain protein with less effect from the polypeptide. On the other hand, there are significant differences in the fluctuations of lysozyme. Both the total fluctuations in the complex and external fluctuation for lysozyme $1 \mathrm{bvkC}$ are larger than those of 2dqjY (Figs. 3a and 3c). The difference between the internal fluctuations for the two lysozymes, $1 \mathrm{bvkC}$ and 2dqjY, is relatively small (Fig. 3b). These results indicate that the Fv fragment and lysozyme are bound more loosely 


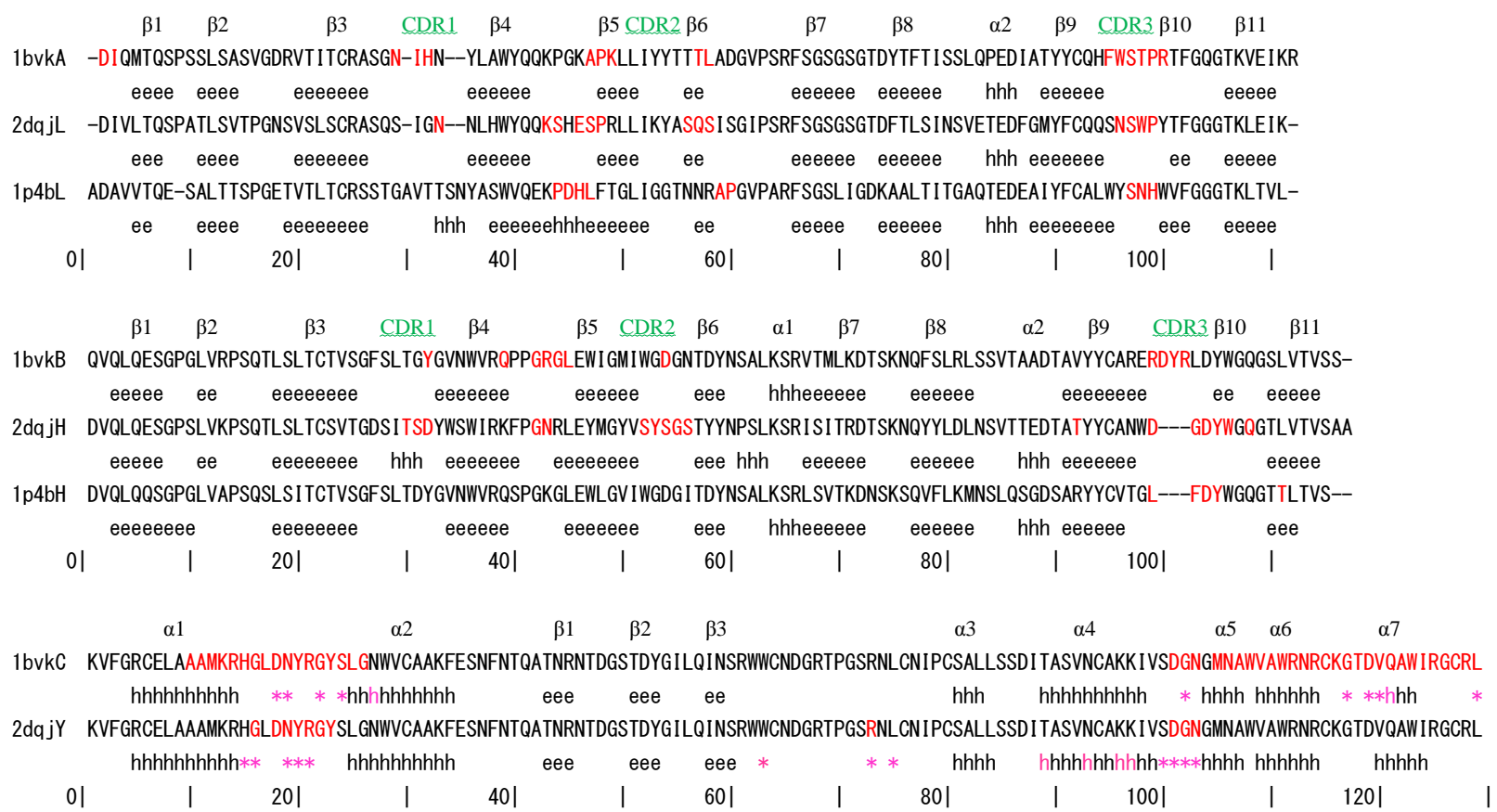

Fig. (2). Multiple structural alignments of three complexes, 1bvk, 2dqj, and 1p4b. The structural alignments of the Fv fragments were performed separately for individual chains at MATRAS web site [35]. The $\alpha$-helix and $\beta$-strand are indicated by letters, h and e, respectively, for each sequence based on the PDBsum [38] and numbered such as $\alpha 1, \alpha 2, \ldots$, and $\beta 1, \beta 2, \ldots$ for referring to them. A complementarity determining region of immunoglobulin is indicated by "CDR". The residue whose cross-correlation between internal and external motions is less than -0.02 is indicated by the red color of the amino acid name in the amino acid sequences. The residues in lysozyme, 1 bvkC, forming hydrogen bonds with antibody residues identified by Holmes et al. [32] are indicated by the red color of the letters, h, e, or an asterisk for the relevant residue. The epitope residues in lysozyme, 2dqjY, defined by Kondo et al. [36] are also indicated in the same way.
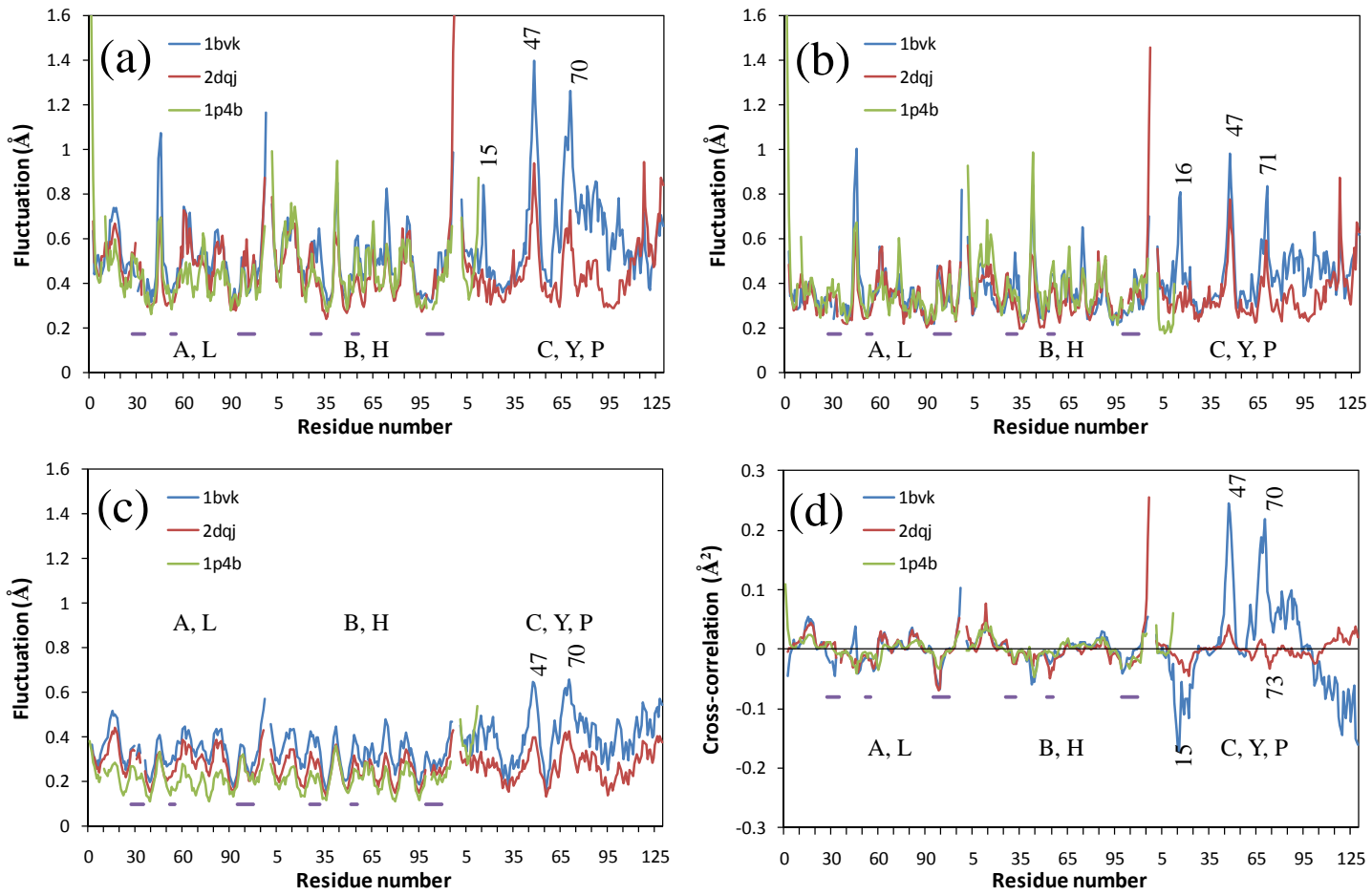

Fig. (3). Fluctuations of atoms and cross-correlation between internal and external motions of three complexes, 1bvk, 2dqj, and 1p4b. The four panels show (a) total fluctuation, $\left\langle\Delta r_{\alpha}^{2}\right\rangle^{1 / 2}$, (b) fluctuation in internal motion, $\left\langle\left(\Delta r_{\alpha}^{\text {int }}\right)^{2}\right\rangle^{1 / 2}$, (c) fluctuation in external motion, $\left\langle\left(\Delta r_{\alpha}^{e x t}\right)^{2}\right\rangle^{1 / 2}$, and (d) cross-correlation between internal and external motions, $\left\langle\Delta r_{\alpha}^{\text {int }} \Delta r_{\alpha}^{\text {ext }}\right\rangle$ averaged by residue. The short line segments indicate CDR positions. The residue numbers of some specific residues are shown for lysozyme. 
(a)

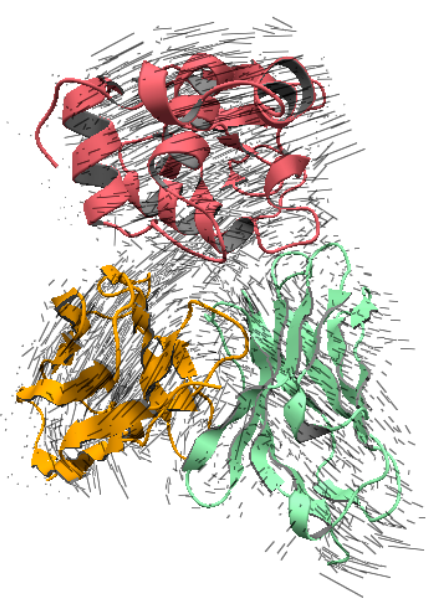

(b)

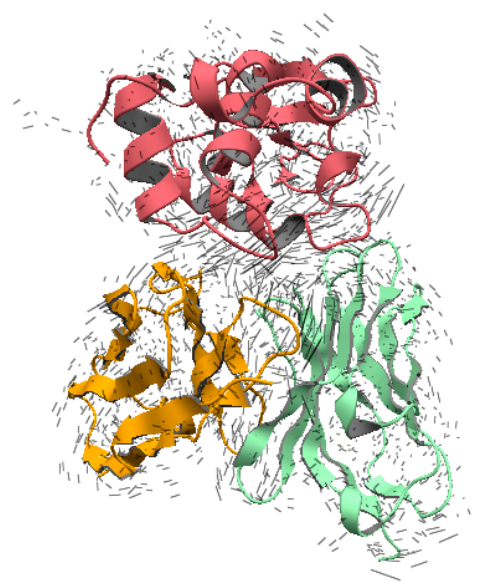

(c)

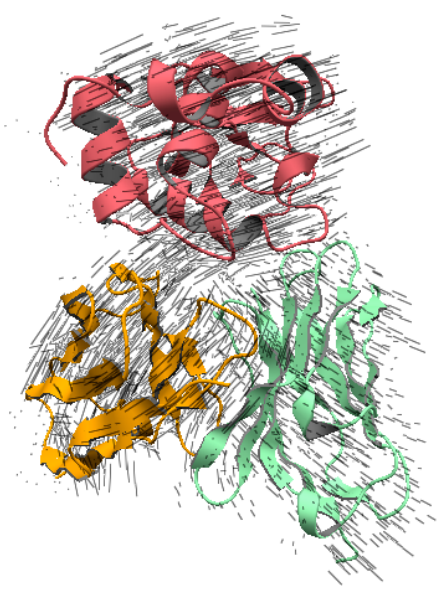

Fig. (4). Example of decomposition of displacement vectors of atoms into internal and external motions. The three types of motions in the lowest-frequency normal mode of 2dqj are shown: (a) total, (b) internal, and (c) external displacement vectors. The vectors calculated at 310 $\mathrm{K}$ are magnified 20 times for clarification. The chains $\mathrm{L}, \mathrm{H}$, and $\mathrm{Y}$ are colored orange, green, and pink, respectively.

in 1 bvk than in 2dqj, and thus the individual chains in 1 bvk can behave more freely and more like a rigid body.

From Fig. (3), we consider that the size of atomic fluctuations in the total motion of the complex reflect the internal motions of individual chains because Fig. (3b) resembles Fig. (3a) more than Fig. (3c) does. However, Fig. (4) provides a different perspective. The directions of the displacement vectors in the total motion of the complex (Fig. 4a) seem well correlated with those in the external motions of the individual chains (Fig. 4c). The same is true for other lower-frequency normal modes of $1 \mathrm{bvk}, 2 \mathrm{dqj}$, and $1 \mathrm{p} 4 \mathrm{~b}$. This suggests that the external motions of the individual chains are mainly responsible for the lower-frequency normal-mode motions of the whole oligomer. The apparent inconsistency between Figs. (3 and $\mathbf{4}$ ), i.e., which motion is dominant in the total motion, internal or external one, can be explained as follows. The directions of the displacement vectors are mainly determined by the external motions. However, the external motion is a rigid body motion and thus the variation in the sizes of the displacement vectors is relatively small as seen in Figs. (3c and $\mathbf{4 c}$ ). On the other hand, the directions and sizes of the displacement vectors for the internal motions are much more mixed. The variation in the sizes of the displacement vectors in the internal motions of the individual chains in Fig. (4b) is more impressive than that in the external motions in Fig. (4c). Specifically, large fluctuations in the internal motion are prominently reflected in the total motion, and thus the fluctuations shown in Figs. (3a) for the total motions and Fig. (3b) for the internal motions seem to have similar features.

Let us consider the fluctuation of lysozyme in more detail. Lysozyme is composed of two domains, $\alpha$-domain (139 and 89-129) and $\beta$-domain (40-88) (Fig. 5). The former is an $\alpha$-helix-rich domain and the latter a $\beta$-sheet-rich one. The helix-loop-helix (HLH) motif (89-114; $\alpha 4-\alpha 6)$ located at the upper lip of the active site and also in the $\alpha$-domain is known to play a key role in antimicrobial function [37]. In the 1bvk complex, lysozyme interacts with the Fv fragment at three regions: a loop between $\alpha$-helices $\alpha 1$ and $\alpha 2$, a loop between $\alpha$-helices $\alpha 4$ and $\alpha 5$ that is also a loop in the HLH motif, and the C-terminal (see Fig. 2). Correspondingly, the large fluctuations occur in the other regions that do not interact with the Fv fragment, e.g., the latter half of the $\beta$-domain and the first $\alpha$-helix $\alpha 4$ in the HLH motif. In particular, the fluctuations of the loops around residues 47 and 70 in the $\beta$ domain are remarkable. They are most remote from the interface with the Fv fragment (see Fig. 7 below). In contrast, lysozyme in 2dqj interacts with the Fv fragment at three different regions in 1bvk: a loop between $\alpha$-helices $\alpha 1$ and $\alpha 2$ that is common to $1 \mathrm{bvk}$, an irregular region around residues 73 and 75 in the $\beta$-domain, and the first $\alpha$-helix $\alpha 4$ in the HLH motif (Fig. 1). The fluctuations are largely suppressed except for the loops around residues 47 and 70 and the Cterminal. While an open-close motion between $\alpha$ - and $\beta$ domains of lysozyme is suppressed by the interaction with the Fv fragment in 2dqj, it is allowed in 1bvk. The fluctuations around residues 35, 52, 62, and 63 involved in a ligand binding pocket located at the interface between the two domains are significantly smaller than for other residues in either complex.

The mean cross-correlations between internal and external fluctuations in Fig. (3d) indicate the angles between internal and external displacement vectors of individual atoms averaged over all normal modes. A negative value means that the internal motion tends to be in the opposite direction of the external motion, and consequently, the total motion of the atom is attenuated. Such a situation is observed at six complementarity determining regions (CDRs) and a loop between $\beta$-strands $\beta 4$ and $\beta 5$ in both light- and heavy-chains of 1 bvk and 2dqj. In addition, the $\alpha$-domain comprising $\mathrm{N}$ and C-terminals of lysozyme in 1bvk has a large negative cross-correlation value. Figs. ( 2 and $\mathbf{3 d}$ ) show that such residues with a large negative value of cross-correlation have 


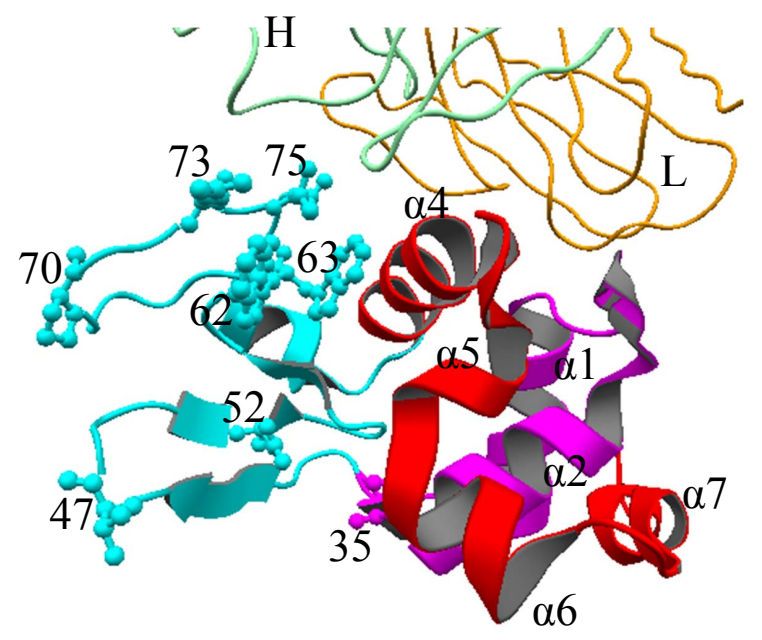

Fig. (5). Three-dimensional structure of lysozyme in 2dqj. Nterminal $\alpha$-domain (1-39), C-terminal $\alpha$-domain (89-129), and $\beta$ domain (40-88) are colored magenta, red, and cyan, respectively. Some residues specified in the text are drawn in a ball-and-stick model with their residue numbers. Parts of chains $\mathrm{L}$ and $\mathrm{H}$ are also shown to see interfaces with lysozyme.

good correspondence with the residues in CDRs in Ig lightand heavy-chains, with the residues forming hydrogen bonds with Ig residues in lysozyme 1 bvkC identified by Holmes et al., [32], and with the epitope residues in lysozyme 2dqjY defined by Kondo et al., [36].

These situations are clearly shown in Fig. (6). The atoms with relatively large negative $\left\langle\Delta r_{\alpha}^{\text {int }} \Delta r_{\alpha}^{\text {ext }}\right\rangle$ values (indicated by the spheres) are localized around the interface regions between the Ig chains and lysozyme. The CDRs at the interface fluctuate to anchor lysozyme and peptide, while the loops between $\beta$-strands $\beta 4$ and $\beta 5$ in light- and heavy-chains of Ig fluctuate to anchor each other chain. In fact, large internal fluctuations of individual Ig chains and lysozyme are localized around the interface regions in the example of Fig. (4b).

In Fig. (7), the translational and rotational vectors of the three lowest-frequency normal modes defined for individual subunits are shown for the three complexes. Their sizes and the angles between them are summarized in Table $\mathbf{1}$.

Although the sizes of the translational vectors cannot be compared with the rotational vectors directly, we can make a rough comparison by using the calculation that the length of an arc of a circle with a radius of $15 \AA$ and subtending an angle of one degree is $0.26 \AA$. In fact, since the radii of gyration of the individual chains are around $13 \AA$, the rotational motions of atoms distant from the center of mass are more dominant than the translational motions.

Interestingly, these vectors of the three normal modes are perpendicular to each other in general. This suggests that various motions are possible by combining them. On the other hand, since the magnitudes of these vectors differ, there are some motions more dominantly involved in actual movement. Such motions characterize the complexes from a dynamic point of view.

For example, while the three rotational vectors in lysozyme of $1 \mathrm{bvk}$ are comparable to each other, the rotational vector of mode 1 is dominant in 2dqj. This fact indicates that while lysozyme is allowed to rotate around various axes that combine the three rotational vectors in 1bvk, it predominantly rotates around the axis of mode 1 perpendicular to the interface with the Fv fragment in 2dqj. On the other hand, the translational vector of the peptide in $1 \mathrm{p} 4 \mathrm{~b}$ is remarkably large in mode 3 . The peptide moves perpendicular
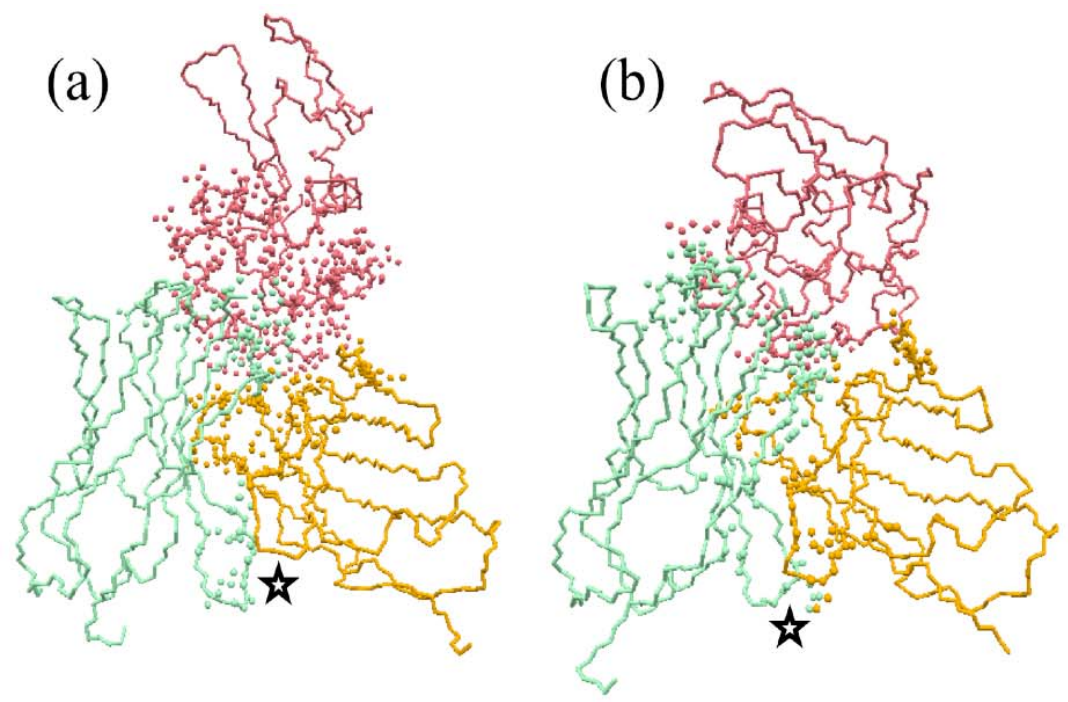

(c)

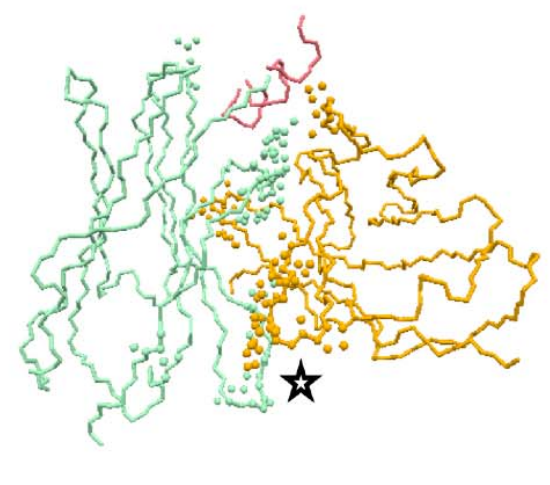

Fig. (6). Atoms with largely negative cross-correlations between internal and external motions. The atoms with $\left\langle\Delta r_{\alpha}^{\text {int }} \Delta r_{\alpha}^{\text {ext }}\right\rangle$ of less than -0.02 are shown by spheres for (a) 1bvk, (b) 2dqj, and (c) 1p4b. The internal motions of these atoms tend to be in the opposite direction of the external motions. The Ig light chains ( $\mathrm{A}$ and $\mathrm{L})$, Ig heavy chains $(\mathrm{B}$ and $\mathrm{H})$, and lysozyme $(\mathrm{C}$ and $\mathrm{Y})$ are colored orange, green, and pink, respectively. The peptide in $1 \mathrm{p} 4 \mathrm{~b}$ is colored pink. The star symbol indicates where the loops between the $\beta$-strands $\beta 4$ and $\beta 5$ in both lightand heavy-chains of immunoglobulin meet each other. 
Table 1. Sizes of Translational and Rotational Vectors of Subunits, Angles Between them, and Motion Types

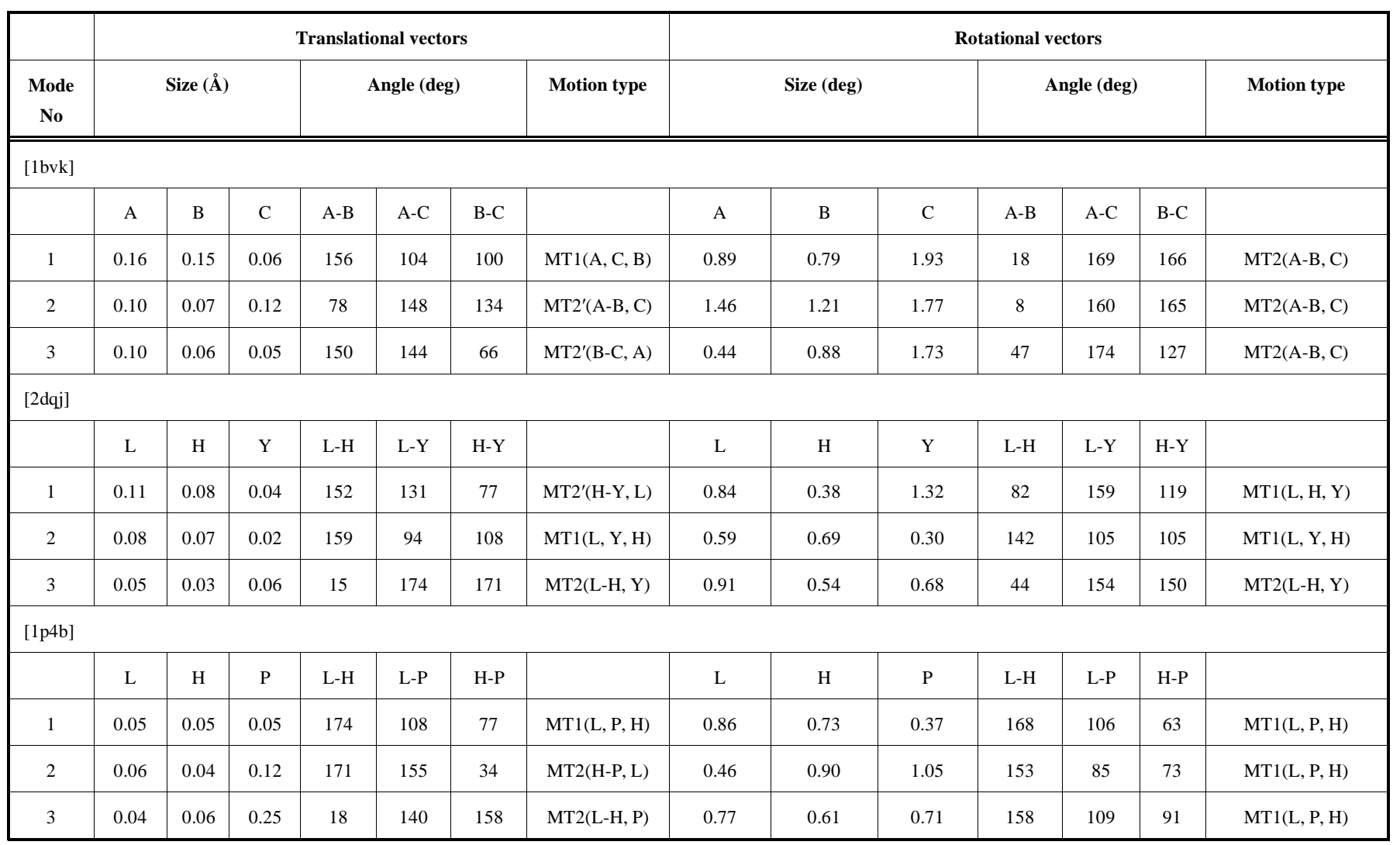

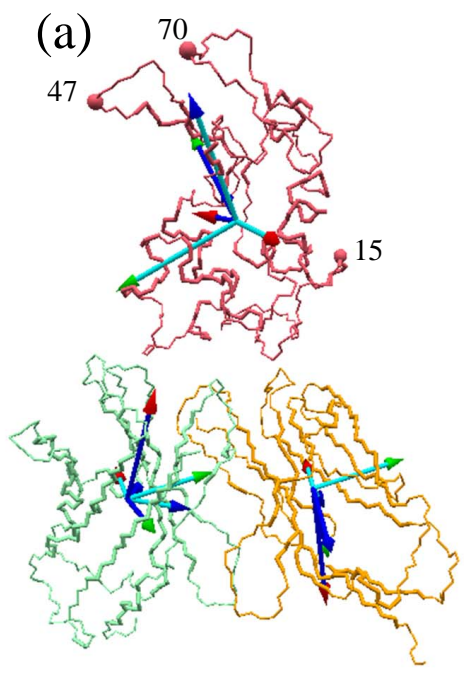

(b)

(c)

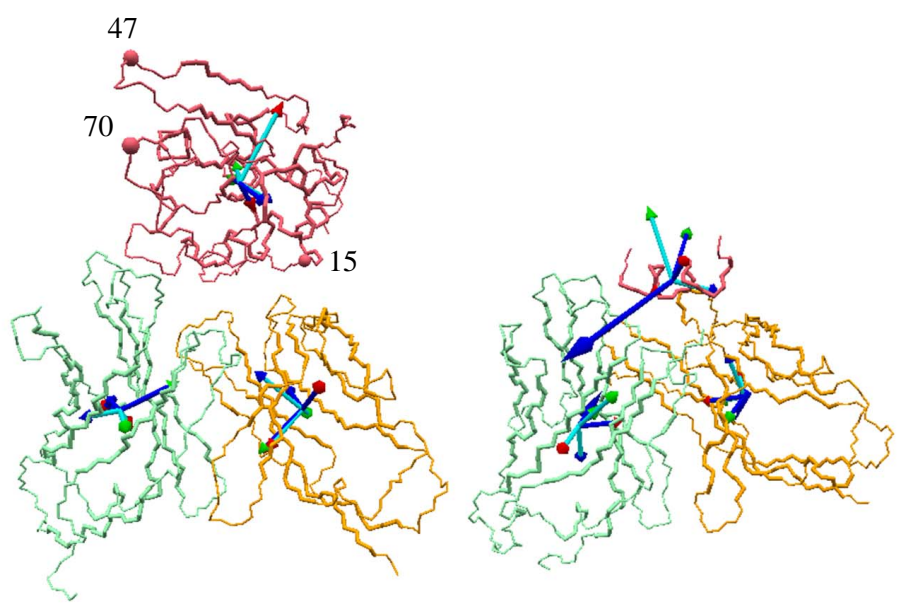

Fig. (7). Translational and rotational vectors of the 3 lowest-frequency normal modes defined for individual subunits: (a) 1bvk, (b) 2dqj, and (c) 1p4b. The arrows with blue and cyan shafts are translational and rotational vectors, respectively. The red, green, and blue arrow heads indicate the first, second, and third lowest-frequency normal modes, respectively. The size of the translational vector is magnified 100 times and the size of the rotational vector (in units of radians) is multiplied by $500 \AA$ rad for clarification. Some specific residues are indicated by spheres and their residue numbers in lysozyme structures (see also Fig. (2) for these residues). For the subunit colors, see the caption of Fig. (6).

to the $\alpha$-helix axis in it and along the interface with the Fv fragment. The translational vectors of the peptide for modes 1 and 2 that are perpendicular to the interface are not very large, but considered to be characteristic motions of the peptide.
The mutual directions of the translational and rotational vectors among the three chains for a given normal mode are also important. The mutual movements of the three chains can be classified into three types based on the results, as shown in Fig. (8). In motion type 1 (MT1), the vectors of 


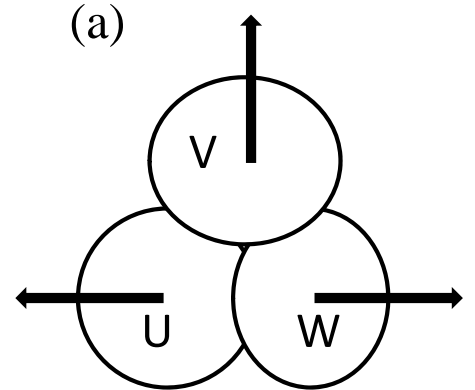

MT1(U, V, W) (b)

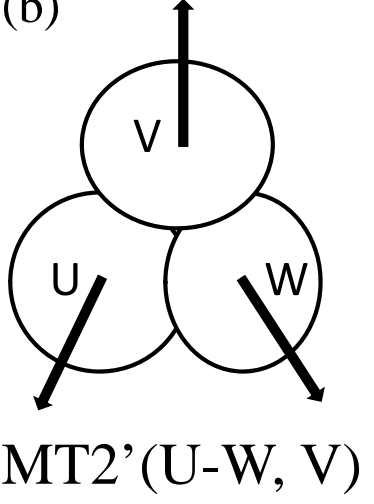

(c)

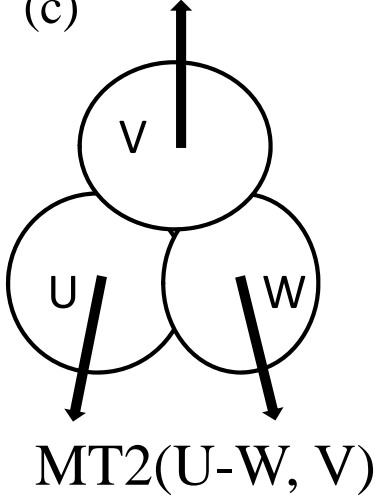

Fig. (8). Three types of mutual motions for subunits. They are defined as follows: Motion type $1, \operatorname{MT} 1(\mathrm{U}, \mathrm{V}, \mathrm{W})=\left\{60^{\circ} \leq \mathrm{Ang}(\mathrm{U}, \mathrm{V})<120^{\circ}\right.$, $\left.60^{\circ} \leq \operatorname{Ang}(\mathrm{V}, \mathrm{W})<120^{\circ}, 120^{\circ} \leq \operatorname{Ang}(\mathrm{U}, \mathrm{W}) \leq 180^{\circ}\right\} ;$ Motion type $2^{\prime}, \mathrm{MT}^{\prime}(\mathrm{U}-\mathrm{W}, \mathrm{V})=\left\{60^{\circ} \leq \operatorname{Ang}(\mathrm{U}, \mathrm{W})<120^{\circ}, 120^{\circ} \leq \mathrm{Ang}(\mathrm{U}, \mathrm{V}) \leq 180^{\circ}\right.$, $\left.120^{\circ} \leq \operatorname{Ang}(\mathrm{W}, \mathrm{V}) \leq 180^{\circ}\right\}$; Motion type 2, MT2(U-W, V) $=\left\{0^{\circ} \leq \operatorname{Ang}(\mathrm{U}, \mathrm{W})<60^{\circ}, 120^{\circ} \leq \operatorname{Ang}(\mathrm{U}, \mathrm{V}) \leq 180^{\circ}, 120^{\circ} \leq \mathrm{Ang}(\mathrm{W}, \mathrm{V}) \leq 180^{\circ}\right\}$, where $U, V$, and $W$ are translational or rotational vectors of the three subunits, and $\operatorname{Ang}(U, V)$ is the angle between vectors $U$ and $V$. It should be noted that the order and the notation of arguments in MT1, MT2', and MT2 has significant meaning for the orientations of the three vectors.

two chains point in the opposite direction to each other and the third vector is perpendicular to them. In motion type 2 (MT2), the vectors of two chains point in similar directions, but the third vector is in the opposite direction. In motion type 2' (MT2'), the vectors of two chains point in roughly similar directions, but further apart than motion type 2, with the third vector in the opposite direction. Although other motion possibilities exist for the chains, only these three types were found for the three lowest-frequency normal modes examined. More precise definitions for these motion types are given in the caption of Fig. (8).

As far as the rotational vector is concerned, the three complexes are well characterized by the above motion types. In 1bvk, any motion types for the three normal modes are MT2, in which the rotational vectors of the light- and heavychains have the same direction and that of the lysozyme has the opposite direction to them. In $1 \mathrm{p} 4 \mathrm{~b}$, any motion types of the three normal modes are MT1, in which the light- and heavy-chains move in the opposite direction to each other and the rotational vector for the peptide is perpendicular to them. The motion types of 2dqj are either MT1 or MT2. This fact suggests that the three chains behave in a more complicated manner than the other complexes because the three chains of 2dqj interact with each other more strongly than 1bvk and the sizes of the three chains are comparable to each other, unlike $1 \mathrm{p} 4 \mathrm{~b}$ (the number of residues are 108,116 , and 129 for A, B, and C chains of 1bvk, respectively; 107, 114, and 129 for L, H, and Y chains of 2dqj, respectively; 110, 112 , and 12 for $\mathrm{L}, \mathrm{H}$, and $\mathrm{P}$ chains of $1 \mathrm{p} 4 \mathrm{~b}$, respectively). $1 \mathrm{p} 4 \mathrm{~b}$ is like a single-chain protein composed of two domains.

In contrast, every complex has a mixture of the motion types for translational vectors probably because the translational motions are not dominant in the external motions. There are interesting common features among the three complexes for the translational motions: $(i)$ every complex has a normal mode of motion type MT1 in which the light chain moves in the opposite direction to the heavy chain while the lysozyme and peptide move in a perpendicular direction to them, and (ii) every complex has a normal mode of motion type MT2 or MT2' in which the light- and heavychains move in similar directions while the lysozyme and peptide move in an opposite direction to them. More detailed movements for these complexes are visualized by animation on the molecular viewer in ProMode-Oligomer.

Finally, the correlations between $\mathrm{C}^{\alpha}$ atom movements averaged over all modes and time for $1 \mathrm{bvk}$ and 2dqj are shown in Fig. (9). Mean correlative motions between the subunits and between the domains in lysozyme appear clearly in these figures. Many positively correlated residue pairs within the Fv fragments (chains A and B of 1bvk, and chains $\mathrm{L}$ and $\mathrm{H}$ in 2dqj) suggest that the residues move together in each of them. In contrast, there are two groups of residues in lysozyme ( $\mathrm{C}$ and $\mathrm{Y}$ chains in 1bvk and 2dqj, respectively) that constitute $\alpha$-domain and $\beta$-domain. While the residues within the same domain move together with positive correlation, the pairs of residues in the different domains move in the opposite direction to each other with negative correlation. This is an open-close motion between $\alpha$ - and $\beta$-domains. As for inter-chain movements, each chain moves in a negatively correlated manner with other chains in general. Particularly, the large negative values between $\beta$ domain in lysozyme and the $\mathrm{Fv}$ fragments are remarkable. More negatively correlated residue pairs in 1bvk than 2dqj result from the fact that the Fv fragment and lysozyme are bound more loosely in 1 bvk than in 2dqj, and thus the individual chains in 1bvk can behave more freely and more like a rigid body as discussed above. This kind of correlation maps are also given for each of the 20 lowest-frequency normal modes in ProMode-Oligomer, and useful for characterizing not only inter-chain motions but also domain motions.

\section{CONCLUSION}

ProMode-Oligomer is a database collecting NMA results for oligomeric proteins and protein-protein complexes. As in 

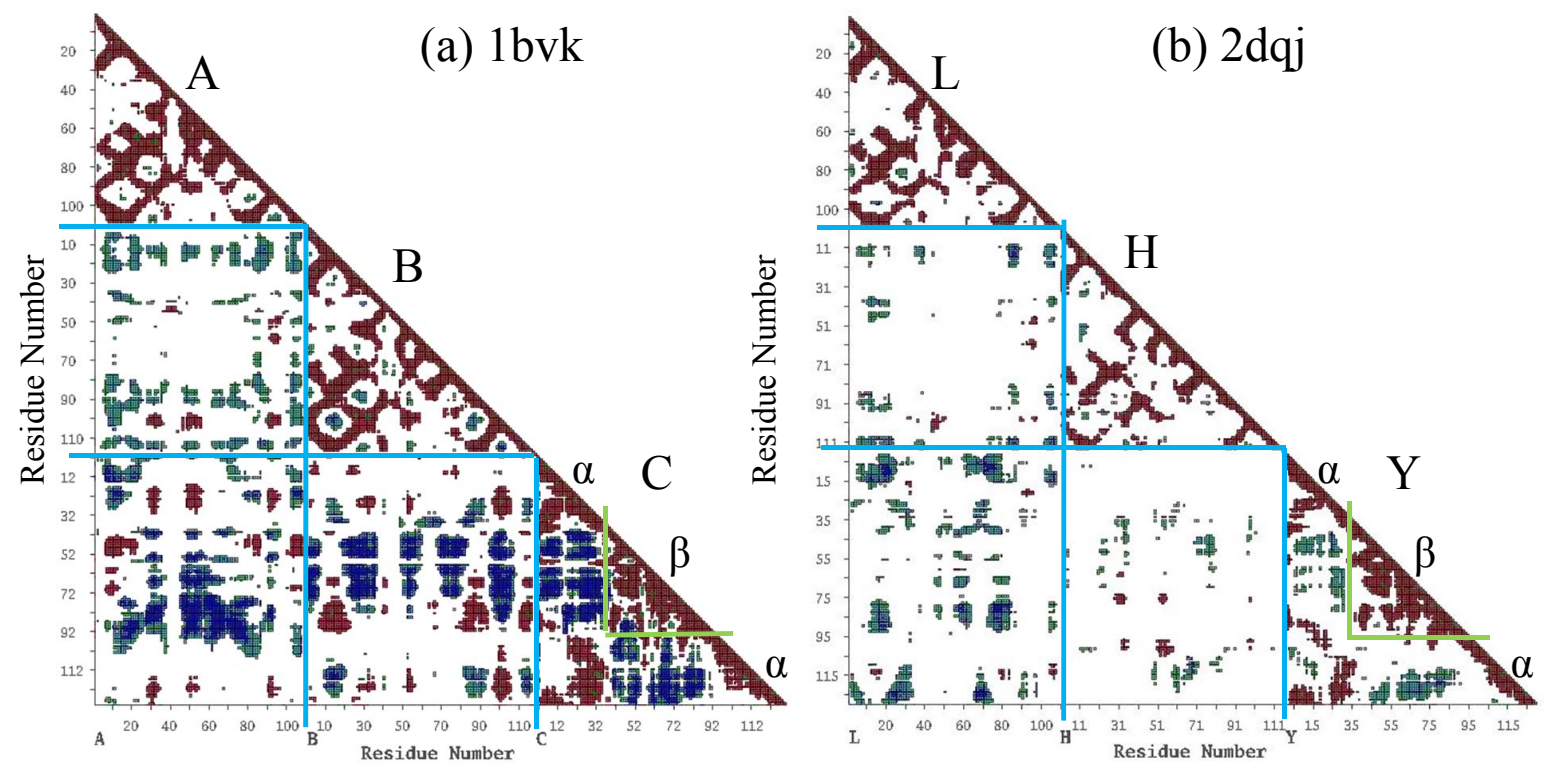

Fig. (9). Correlation maps for (a) 1bvk and (b) 2dqj. Correlations between C $\alpha$ atom movements averaged over all modes and time are shown in the triangular maps. The correlation is defined as a normalized inner product of the normal mode displacement vectors of two C $\alpha$ atoms. Each residue pair is assigned a color according to its correlation value: brown ( $>0.3)$, red $(0.3$ to 0.2$)$, white $(0.2$ to -0.2$)$, green blue $(-0.2$ to $-0.3)$, and blue $(<-0.3)$. The constituent chains and the $\alpha$ - and $\beta$-domains in lysozyme are indicated.

the ProMode database for monomers and individual subunits of oligomers, NMA was performed for a full-atom system using dihedral angles as independent variables, and the results (fluctuations of atoms, fluctuations of dihedral angles, correlations between atomic fluctuations, etc.) were released on the web. The visualization of vibrating oligomers by animation on a molecular viewer is helpful for creating an image of the dynamic structure of a protein. In addition, a decomposition of displacement vectors into internal and external ones makes it possible to provide information about the mutual movements of the subunits, such as translational and rotational motions, and also about the characteristic relationship between the internal and external motions of atoms around an interface region. In this paper, what we have learned in ProMode-Oligomer is illustrated, with the Fv fragments forming complexes with lysozyme and with a 12residue peptide. Additionally, a comparative study has been shown to be more fruitful from this analysis.

Protein oligomerization is a remarkable strategy for producing functional divergence in protein evolution. Threedimensional structures of oligomeric proteins have been studied to reveal oligomerization mechanisms, paying particular attention to their subunit-subunit interface regions. However, there are many varieties of oligomerization behaviors, and thus it can be difficult to find not only common features, but also some aspects useful for characterizing individual oligomers. In such a situation, it is important to investigate them from various perspectives. The dynamic features of their three-dimensional structures should contribute to solving this problem. ProMode-Oligomer can be a useful first step towards understanding the dynamic structures of oligomeric proteins and protein-protein complexes. In this manner, we are now investigating the interfaces of dimeric proteins by NMA to characterize them from a dynamic point of view with the results to be published later.
Since it is difficult to exhaustively perform NMA on PDB data due to the CPU-intensive energy minimization required in a framework of orthodox molecular mechanics, only a small number of results has been released at ProMode-Oligomer. In order to overcome this problem, the elastic-network-model (ENM)-based NMA is a promising approach for performing NMA on PDB data exhaustively, although it is still important to perform NMA in the orthodox manner. We are developing an ENM-based NMA program using dihedral angles as independent variables. With this program we are hoping to perform computations for all atoms in any molecule in the PDB to take advantage of the smaller number of independent variables required. These results will also be released on the Web.

\section{ACKNOWLEDGEMENTS}

Development of ProMode-Oligomer is part of a project for the advancement of the PDBj (Director: Professor Haruki Nakamura, Osaka University) that is supported by grant-inaid from the Institute for Bioinformatics Research and Development, Japan Science and Technology Agency (BIRDJST) and the Ministry of Education, Culture, Sports, Science and Technology (MEXT).

\section{CONFLICT OF INTEREST}

None Declared.

\section{REFERENCES}

[1] N. Gō, T. Noguti, and T. Nishikawa, "Dynamics of a small globular protein in terms of low-frequency vibrational modes", Proc. Natl. Acad. Sci. USA, vol. 80, pp. 3696-3700, 1983.

[2] M. Levitt, C. Sander, and P.S. Stern, "Protein normal-mode dynamics: Trypsin inhibitor, crambin, ribonuclease and lysozyme”, $J$. Mol. Biol., vol. 181, pp. 423-447, 1985.

[3] M.M. Tirion, "Large amplitude elastic motions in proteins from a single-parameter, atomic analysis”, Phys. Rev. Lett., vol. 77, pp. 1905-1908, 1996. 
[4] I. Bahar, A.R. Atilgan, and B. Erman, "Direct evaluation of thermal fluctuations in proteins using a single-parameter harmonic potential”, Fold Des., vol. 2, pp. 173-181, 1997.

[5] K. Hinsen, "Analysis of domain motions by approximate normal mode calculations”, Proteins, vol. 33, pp. 417-429, 1998.

[6] F. Tama and Y.-H. Sanejouand, "Conformational change of proteins arising from normal mode calculations”, Protein Eng., vol. 14, pp. 1-6, 2001.

[7] W.G. Krebs, V. Alexandrov, C.A. Wilson, N. Echols, H. Yu, and M. Gerstein, "Normal mode analysis of macromolecular motions in a database framework: Developing mode concentration as a useful classifying statistic”, Proteins, vol. 48, pp. 682-695, 2002.

[8] N. Reuter, K. Hinsen, and J.-J. Lacapère, "Transconformations of the SERCA1 Ca-ATPase: A Normal Mode Study”, Biophys. J., vol. 85, pp. 2186-2197, 2003.

[9] V. Alexandrov, U. Lehnert, N. Echols, D. Milburn, D. Engelman, and M. Gerstein, "Normal modes for predicting protein motions: A comprehensive database assessment and associated web tool", Protein Sci., vol. 14, pp. 633-643, 2005.

[10] I. Bahar and A.J. Rader, "Coarse-grained normal mode analysis in structural biology”, Curr. Opin. Struct. Biol., vol. 25, pp. 1-7, 2005.

[11] D. Tobi and I. Bahar, "Structural changes involved in protein binding correlate with intrinsic motions of proteins in the unbound state”, Proc. Natl. Acad. Sci. USA, vol. 102, pp. 18908-18913, 2005.

[12] S.E. Dobbins, V.I. Lesk, and J.E. Sternberg, "Insights into protein flexibility: The relationship between normal modes and conformational change upon protein-protein docking”, Proc. Natl. Acad. Sci. USA, vol. 105, pp. 10390-10395, 2008.

[13] H. Wako and S. Endo, "Ligand-induced conformational change of a protein reproduced by a linear combination of displacement vectors obtained from normal mode analysis”, Biophys. Chem., vol. 159, pp. 257-266, 2011.

[14] D.J. Jacobs, A.J. Rader, L.A. Kuhn, and M.F. Thorpe, "Protein flexibility predictions using graph theory”, Proteins, vol. 44, pp. 150-165, 2001.

[15] S. Hirose, K. Yokota, Y. Kuroda, H. Wako, S. Endo, S. Kanai, and T. Noguchi, "Prediction of protein motions from amino acid sequence and its application to protein-protein interaction", $B M C$ Struct. Biol., vol. 10, no. 20, pp. 1-18, 2010.

[16] A. Bornot, C. Etchebest, and A.G. De Brevern, "Predicting protein flexibility through the prediction of local structures”, Proteins, vol. 79, pp. 839-852, 2011.

[17] H. Wako, M. Kato, and S. Endo, "ProMode: A database of normal mode analyses on protein molecules with a full-atom model”, Bioinformatics, vol. 20, pp. 2035-2034, 2004.

[18] K. Suhre and Y.H. Sanejouand, "elNémo: A normal mode webserver for protein movement analysis and the generation of templates for molecular replacement”, Nucleic Acids Res., vol. 32, pp. W610-W614, 2004.

[19] L.W. Yang, X. Liu, C.J. Jursa, M. Holliman, A.J. Rader, H.A. Karimi, and I. Bahar, " $i$ GNM: A database of protein functional motions based on Gaussian network model”, Bioinformatics, vol. 21, pp. 2978-2987, 2005.

[20] S.M. Hollup, G. Salensminde, and N. Reuter, "WEBnm@: A web application for normal mode analyses of proteins", BMC Bioinformatics, vol. 6, pp. 1-8, 2005.

[21] L.W Yang, A.J. Rader, X. Liu, C.J. Jursa, S.C. Chen, H.A. Karimi, and I. Bahar, "oGNM: Online computation of structural dynamics using the Gaussian network model”, Nucleic Acids Res., vol. 34, pp. W24-W31, 2006.
[22] E. Lindahl, C. Azuara, P. Koehl, and M. Delarue, "NOMAD-Ref: Visualization, deformation and refinement of macromolecular structures based on all-atom normal mode analysis", Nucleic Acids Res., vol. 34, pp. W52-W56, 2006.

[23] Y. Seno and N. Gō, "Deoxymyoglobin studied by the conformational normal mode analysis: I. Dynamics of globin and the hemeglobin interaction”, J. Mol. Biol., vol. 216, pp. 95-109, 1990.

[24] Y. Seno and N. Gō, "Deoxymyoglobin studied by the conformational normal mode analysis: II. The conformational change upon oxygenation”, J. Mol. Biol., vol. 216, pp. 111-126, 1990.

[25] M.Y. Niv and M. Filizola, "Influence of oligomerization on the dynamics of G-protein coupled receptors as assessed by normal mode analysis”, Proteins, vol. 71, pp. 575-586, 2008.

[26] I. Bahar, T.R. Lezon, A. Bakan, and I.H. Shrivastava, "Normal mode analysis of biomolecular structures: functional mechanisms of membrane proteins”, Chem. Rev., vol. 110, pp. 1463-1497, 2010.

[27] H. Ishida, Y. Jochi, and A. Kidera, "Dynamic structure of subtilisin-eglin c complex studied by normal mode analysis", Proteins, vol. 32, pp. 324-333, 1998.

[28] C. Eckart, "Some studies concerning rotating axes and polyatomic molecules”, Phys. Rev., vol. 47, pp. 552-558, 1935.

[29] H. Wako, S. Endo, K. Nagayama, and N. Gō, "FEDER/2: Program for static and dynamic conformational energy analysis of macromolecules in dihedral angle space”, Comp. Phys. Comm., vol. 91, pp. 233-251, 1995.

[30] G. Nemethy, K.D. Gibson, K.A. Palmer, C.N. Yoon, G. Paterlini, A. Zagari, S. Rumsey, and H.A. Scheraga, "Energy parameters in polypeptides. 10. Improved geometric parameters and nonbonded interactions for use in the ECEPP/3 algorithm, with application to proline-containing peptides”, J. Phys. Chem., vol. 96, pp. 64726484, 1992.

[31] H. Wako and N. Gō, "Algorithm for rapid calculation of Hessian of conformational energy function of proteins by supercomputer”, $J$. Comp. Chem., vol. 8, pp. 625-635, 1987.

[32] M.A. Holmes, T.N. Buss, and J. Foote, "Conformational correction mechanisms aiding antigen recognition by a humanized antibody", J. Exp. Med., vol. 187, pp. 479-485, 1998.

[33] M. Shiroishi, K. Tsumoto, Y. Tanaka, A. Yokota, T. Nakanishi, H. Kondo, and I. Kumagai, "Structural consequences of mutations in interfacial Tyr residues of a protein antigen-antibody complex", $J$. Biol. Chem., vol. 282, pp. 6783-6791, 2007.

[34] C. Zahnd, S. Spinelli, B. Luginbühl, P. Amstutz, C. Cambillau, and A. Plückthun, "Directed in vitro evolution and crystallographic analysis of a peptide-binding single chain antibody fragment (scFv) with low picomolar affinity", J. Biol. Chem., vol. 279, pp. 1887018877, 2004.

[35] T. Kawabata, "MATRAS: A program for protein 3D structure comparison”, Nucleic Acids Res., vol. 31, pp. 3367-3369, 2003.

[36] H. Kondo, M. Shiroishi, M. Matsushima, K. Tsumoto, and I. Kumagai, "Crystal structure of anti-hen egg white lysozyme antibody (HyHEL-10) Fv-antigen complex local structural changes in the protein antigen and water-mediated interactions of $\mathrm{Fv}$-antigen and light chain-heavy chain interfaces”, J. Biol. Chem., vol. 274, pp. 27623-27631, 1999.

[37] H.R. Ibrahim, U. Thomas, and A. Pellegrini, "A helix-loop-helix peptide at the upper lip of the active site cleft of lysozyme confers potent antimicrobial activity with membrane permeabilization action”, J. Biol. Chem., vol. 276, pp. 43767-43774, 2001.

[38] R.A. Laskowski, "PDBsum new things", Nucleic Acids Res., vol. 37, pp. D355-D359, 2009.

(C) Wako and Endo; Licensee Bentham Open.

This is an open access article licensed under the terms of the Creative Commons Attribution Non-Commercial License (http://creativecommons.org/licenses/by-nc/3.0/) which permits unrestricted, non-commercial use, distribution and reproduction in any medium, provided the work is properly cited. 